\title{
Difficulties on Emotion Regulation Predict Mental Health of Coronary Heart Disease Patients in Cardiac Rehabilitation but Heart Rate Variability does Not
}

\author{
Elise Batselée,2*, Sophie Vanden Eynde ${ }^{3}$, Nathalie Egée ${ }^{3}$, Michel Lamotte ${ }^{3}$, Philippe Vande Borne ${ }^{3}$ and \\ Carole Fantini-Hauwel ${ }^{3}$
}

${ }^{1}$ Department of Clinical Psychology, University of Brussels, Belgium

${ }^{2}$ National Fund for Scientific Research, Belgium

${ }^{3}$ Erasme Hospital, Belgium

*Corresponding author: Elise Batselé, Department of Clinical Psychology, University of Brussels, Belgium.

Received Date: August 30, 2019

Published Date: September 04, 2019

\begin{abstract}
Purpose: Depression and anxiety have been extensively associated with adverse outcomes in coronary heart disease (CHD) patients. However, psychological and physiological processes underlying the persistence of these troubles in CHD patients attending cardiac rehabilitation are poorly investigated. Emotion regulation difficulties and heart rate variability (HRV) could be some of these processes. Thus the aim of this study was to assess the predictive value of emotion regulation difficulties and HRV on depression and anxiety symptoms persistence in CHD patients.
\end{abstract}

Methods: Eigthy-four patients who recently presented a myocardial infarction were evaluated at the beginning of cardiac rehabilitation. Fourty-two patients continued their rehabilitation program and were then assessed three months later. They completed the Difficulties in Emotion Regulation Scale (DERS), the Hospital Anxiety and Depression scale (HADS) and underwent a five minutes resting HRV measure.

Results: Emotion regulation difficulties predicted depression and anxiety persistence at 3-month follow-up even after controlling for depression or anxiety scores at baseline. Contrary to our expectations, HRV did not predict depression or anxiety symptoms persistence and was not associated with emotion regulation difficulties.

Conclusion: This study highlights the predictive value of emotion regulation difficulties on the persistence of depression and anxiety symptoms at the end of cardiac rehabilitation of CHD patients. However, HRV was not associated with either depression or anxiety supporting the idea of mixed litterature and calling for future research.

Keywords: Heart rate variability; Emotion regulation; Depression; Anxiety; Coronary heart disease

Abbreviations: HRV: Heart rate variability; CHD: Coronary heart disease

\section{Introduction}

Emotion regulation encompasses the processes by which individuals shape the trajectory of one or more component(s) of an emotional response. Regulation strategies can impact the type, intensity, time course, and quality of emotions [1]. It requires to (1) be aware and understand emotions, (2) accept emotions, (3) be able to control impulsive behaviors and behave in accordance with desired goals when experiencing negative emotions, and (4) be able to use situationally appropriate emotion regulation strategies to meet individual goals and environmental demands. The lack of any or all of these abilities indicates the presence of difficulties in emotion regulation [2]. Emotion regulation may be intrapersonal (regulating one's own emotions) or interpersonal (regulating someone else's emotions) [3]. Difficulties in intrapersonal emotion regulation may be conceptualized as a transdiagnostic factor of psychopathology and have been widely associated with depression and anxiety $[4,5]$. Indeed, depressed individuals usually report a more frequent use of dysfunctional strategies (e.g., emotion suppression, rumination, catastrophizing) and a less frequent use of functional strategies (e.g., reappraisal, self-disclosure) $[6,7,8,9]$. They also exhibit less understanding and clarity of their emotions 
[9] and to be less acceptant of negative feelings [6]. Furthermore, some researchers have suggested that difficulties in emotion regulation are not only concomitant of depression but also a risk factor for repeated depression' episodes [10,11,12,9]. There is also emerging evidence that difficulties in emotion regulation is closely related to anxiety and its pathogenesis $[13,14,15]$. Indeed, individuals suffering from anxiety experience and express more emotional intensity, have more difficulties identifying and understanding emotions, have difficulties accepting emotions, and exhibit more difficulties in using effective regulation strategies [13]. These emotion regulation difficulties would also contribute to the maintenance of the disorder [14]. If the links between difficulties in emotion regulation and mental health seem well established in depressive and anxious populations [5], very few studies have examined the impact of these difficulties in coronary heart disease (CHD) patients. This lack of investigation is surprising because literature has extensively shown the important prevalence of depression and anxiety in this population [16]. Furthermore, these psychological disorders have deleterious impact on the prognostic of CHD patients $[17,18]$. Thus, it seems crucial to understand the processes that may account for the maintenance of depression and anxiety in these patients.

One physiological process that is both associated with emotion regulation and depression as well as anxiety is heart rate variability (HRV) $[19,20]$. HRV is defined as the time intervals between each heartbeat and reflects the autonomic nervous system (ANS) function [21]. HRV has been first investigated in cardiology as it appears to be an important and independent prognostic factor in CHD [22]. Furthermore, a decreased HRV has been advanced as a possible physiological process explaining the link between depression or anxiety and mortality in cardiovascular diseases [23,24]. Indeed, Thayer \& Lane neurovisceral integration model [21] has suggested that HRV is a biomarker of auto-regulation processes when emotions arise as it measures the balance between the sympathetic nervous system (SNS) and the parasympathetic nervous system (PNS). Decreased HRV is thought to reflect depressed vagal modulation from the PNS associated with an increased of the SNS activation. When emotions arise the PNS allows rapid withdrawal of the vagal brake that promotes transitory mobilization of the SNS to adapt to the environment [25]. In this idea, a recent meta-analysis has shown strong evidence that HRV is associated with top-down self-regulatory processes including executive functioning, emotion regulation, working memory, attention regulation [26]. All these processes are involved in both depression and anxiety disorders $[27,28,29]$. Thus, it is possible to hypothesize that a decreased HRV explain the relationships between emotion regulation difficulties and mental Health [30]. It is to note that HRV could also be a moderator of this relationship as it has already been shown that HRV can have a buffer effect for deleterious consequences of personality traits or dysfunctional regulation strategies $[31,32,33]$.

Thus, the aim of this study is to examine the interaction between emotion regulation difficulties and HRV and their combined or individual effects on depression and anxiety symptoms persistence in CHD patients. With respect to the mixed results observed in the literature, two different models will be tested. The first one suggests that emotion regulation difficulties at baseline will predict persistence of depression and anxiety symptoms at follow-up but that this relationship is moderated by HRV's level at baseline. The second one posits that emotion regulation difficulties at baseline will predict depression and anxiety persistence at follow-up but that this relationship is mediated by HRV's level at baseline.

\section{Method}

\section{Population}

This study is part of a larger study designed to assess the predictive value of global emotions management, here indexed by difficulties in emotion regulation, and HRV on mental and somatic health of CHD patients involved in cardiac rehabilitation. Eightyfour patients suffering from a myocardial infarction were recruited in a belgian hospital (mean age $=61.94 \pm 8.90 ; 17$ females). On this initial pool of patients, $50 \%$ (42 patients) withdrawed from cardiac rehabilitation which is a major problem in Europe as only $33 \%$ to $56 \%$ adhere to the cardiac rehabilitation until the end of the program [34]. Thus, we have follow-up data for 42 patients (mean age $=61.38 \pm 8.86$; 8 females). Regarding education level, $37.8 \%$ of the sample reached university while $45.1 \%$ completed high school and $17.1 \%$ stopped their studies in primary school. Patients were included based on their medical diagnosis and drugs prescription. Only patients with medically diagnosed myocardial infarction and consuming beta-blockers were recruited. Exclusion criteria were consuming tricyclic antidepressant drugs as it impacts HRV [35], presenting a severe psychiatric disorder (such as psychotic troubles), having difficulties in understanding and speaking French language, and demonstrating an apparent cognitive deficit that could impact questionnaires completion. Written informed consent was obtained from all patients. The hospital's ethic committee approved this study.

\section{Procedure}

Patients were recruited in the cardiac rehabilitation unit of a Belgian hospital at the beginning of their program (between the first and third session). The first evaluation is referred as Time 1 in the rest of the manuscript. Patients were systematically tested between 9:00 and 12:00 am in a medical consultation local of the cardiac rehabilitation unit. The total duration of the study was 45 minutes. Patients first fulfilled socio-demographic and psychological questionnaires and then underwent a resting HRV measure during 6 minutes in order to extract 5 valid minutes. Resting HRV was measured using the Polar® V800 that has been validated as comparable to an electrocardiograph. During this measure, participants were seated spontaneously breathing and were instructed not to move. Three month after the inclusion, patients were tested again using the same procedure. This second evaluation is referred as Time 2 in the rest of the article. 


\section{Instruments}

Emotion regulation difficulties: Difficulties in emotion regulation scale (DERS) is a 36-item questionnaire scored on a 5-point Likert scale ranging from 1 (almost never) to 5 (almost always) [2]. One total score and 6 sub-scores are computed a) nonacceptance of emotional responses (NONACCEPT) b) difficulties in engaging in goal-oriented behaviours when experience negative emotions (GOALS) c) difficulties in controlling impulsive behaviours when experiencing negative emotions (IMPULSE) d) lack of emotional awareness (AWARE) e) lack of strategies to regulate emotions (STRATEGIES) f) lack of emotional clarity (CLARITY). At Time 1, the total DERS score exhibited a good internal consistency with $\alpha=.90$, as well as for most of its subdimensions with the exception for clarity and goals to a lesser extent (NONACCEPT $\alpha=$ .84 ; GOALS $\alpha=.65$; IMPULSE $\alpha=.77$; AWARE $\alpha=.75$; STRATEGIES $\alpha=.84$; CLARITY $\alpha=.50$ ). We did not exhibit the data for the DERS at Time 2 because it was highly correlated with the DERS at Time 1 $(r=.85, \mathrm{p} \leq .01)$ suggesting that the DERS is a trait measure of stable emotion regulation difficulties.

Depression and anxiety: The French validated version of the Hospital Anxiety and Depression Scale [36] was used to assess anxious and depressive symptoms. This is a 14-item scale (7 for anxiety and 7 for depression) scored on a 4-point Likert scale ranging from 0 to 3 . The HADS exhibited good internal consistency at time 1 (depression $\alpha=.71$, anxiety $\alpha=.70$ ) At time 2, depression score had also good internal consistency $(\alpha=.70)$ but the consistency of anxiety score was questionable $(\alpha=.60)$.

Heart rate variability: HRV was measured using a Polar ${ }^{\circ}$ V800 heart rate monitor allowing to extract HRV parameters. The electrode belt was dampened and placed according to Polar's guidelines, tightly but comfortably just below the chest muscles. Measurements were conducted during 6 minutes (spontaneous breathing and resting state) to be able to extract 5 valid minutes, as recommended by the [37]. The variability between successive R-spikes was then analyzed using Kubios 3.0.2 premium version [38], which allows for the calculation of time- and frequencydomain indices of HRV. Artifacts within the R-R series were visually detected, and we applied an automatic artifact correction level [38]. Among the HRV indices, we chose to use the RMSSD (ms2), a time domain measure of HRV reflecting the PNS modulation of heart rate [37]. RMSSD is thought to be relatively free of respiratory influences and thus appear as a more reliable measure of HRV than others indices [39,37]. The RMSSD has been natural log-transformed (ln) to fit assumptions of linear analyses [40].

\section{Statistical analyses}

All statistical analyses were computed using the software package SPSS version 25 [47]. To identify potential covariates to include in subsequent analyses, Pearson correlations between age and the dependent variables (depression and anxiety scores at Time 2) were computed. One-way ANOVA's were also used to test differences between genders on the dependent variables. Relationships between emotion regulation difficulties, depression and anxiety symptoms, and HRV overtime were examined using Pearson correlations. Logistic regression analyze was used to examine group differences in cardiac rehabilitation dropout. Cardiac rehabilitation dropout was coded as follow: $1=$ patients who stopped the program and $0=$ patients who continued the program. The model included five predictors: age, depression, anxiety, total DERS score, and HRV. Moderation (model 1) and mediation (model 4) analyses were performed using the PROCESS macro with 10.000 bootstrap samples. We first used the total DERS score before conducting the same analyses with each subdimension of this scale a part as our sample is too small to put all the subdimensions in the same analyze. Analyses were computed as follow:

a) Anxiety symptoms at Time 2 as outcome variable, DERS scores at Time 1 as independent variable, HRV at Time 1 as mediator or moderator, and anxiety symptoms at Time 1 as covariable.

b) Depression symptoms at Time 2 as outcome variable, DERS scores at Time 1 as independent variable, HRV at Time 1 as mediator or moderator, and depression symptoms at Time 1 as covariable.

\section{Result}

\section{Dropouts analyze}

We used logistic regression to assess group differences in cardiac rehabilitation dropout. The model including age, depression, anxiety, the total DERS score, and HRV was not significant $(\chi 2(5)=$ $3.41, \mathrm{~ns}$ )

\section{Covariates analyses}

Age did not correlate with either depression or anxiety at Time 2 (respectively $r=-.07$, ns ; r= $-.19 \mathrm{~ns}$ ). One-way ANOVAs did not reveal any significant differences on depression or anxiety at Time 2 depending of gender (respectively $\mathrm{F}(1,41)=.44, \mathrm{p}=.51 ; \mathrm{F}(1,41)$ $=.48, \mathrm{~ns})$. Education level did not influence depression $(\mathrm{F}(4,35)=$ $.98, \mathrm{~ns})$ or anxiety $(\mathrm{F}(4,35)=4.59, \mathrm{~ns})$ at Time 2 . Therefore, these factors were not entered as covariates in subsequent analyses. Descriptives statistics and the time course of changes in HRV levels, depression and anxiety symptoms are summarized in Table 1.

Table 1: Descriptives Statistics.

\begin{tabular}{|c|c|c|c|}
\hline & Time 1 & Time 2 & \\
\hline Variables & Mean (SE) & Mean (SE) & t \\
\hline Age & $61.38(8.86)$ & - & - \\
\hline DERS Total & $82.82(20.47)$ & - & - \\
\hline NONACCEPT & $13.89(5.44)$ & - & - \\
\hline GOALS & $12.96(3.96)$ & - & - \\
\hline IMPULSE & $11.78(4.80)$ & - & - \\
\hline AWARE & $16.22(4.68)$ & - & - \\
\hline STRATEGIES & $17.04(6.72)$ & - & -1.58 \\
\hline CLARITY & $10.93(3.42)$ & - & 0.8 \\
\hline HRV & $2.93(.80)$ & $3.12(.91)$ & 0.13 \\
\hline Depression & $4.48(3.37)$ & $4.19(3.23)$ & $7.45(3.04)$ \\
\hline Anxiety & $7.50(3.18)$ & & \\
\hline
\end{tabular}


Correlation analyses: At Time 1, depression and anxiety correlated moderately with the total DERS score (respectively $r=$ $.46, \mathrm{p} \leq .01$ and $\mathrm{r}=.59, \mathrm{p} \leq .01$ ). All the subdimensions of the DERS at Time 1, except AWARE, correlated with depression and anxiety at Time 1 as detailed in Table 2. Depression and anxiety at Time 1

Table 2: Correlation matrix

\begin{tabular}{|c|c|c|c|c|c|c|c|c|c|c|c|c|}
\hline & $\mathbf{2}$ & $\mathbf{3}$ & $\mathbf{4}$ & $\mathbf{5}$ & $\mathbf{6}$ & $\mathbf{7}$ & $\mathbf{8}$ & $\mathbf{9}$ & $\mathbf{1 0}$ & $\mathbf{1 1}$ & $\mathbf{1 2}$ & $\mathbf{1 3}$ \\
\hline 1. HRV T1 & 0.04 & 0.05 & 0.04 & 0.04 & 0.12 & -0.03 & -0.07 & 0.08 & 0.03 & $.58^{* *}$ & 0.11 & -0.01 \\
\hline 2. DEP T1 & - & $.59^{* *}$ & $.46^{* *}$ & $.22^{*}$ & $.39^{* *}$ & $.30^{* *}$ & 0.13 & $.48^{* *}$ & $.37^{* *}$ & 0.01 & $.75^{* *}$ & $.46^{* *}$ \\
\hline 3. ANX T1 & - & - & $.59^{* *}$ & $.48^{* *}$ & $.48^{* *}$ & $.41^{* *}$ & 0.03 & $.62^{* *}$ & $.38^{* *}$ & 0.21 & $.53^{* *}$ & $.73^{* *}$ \\
\hline 4. DERS T1 & - & - & - & $.79^{* *}$ & $.78^{* *}$ & $.84^{* *}$ & 0.21 & $.85^{* *}$ & $.69^{* *}$ & 0.19 & $.56^{* *}$ & $.61^{* *}$ \\
\hline 5. NONA T1 & - & - & - & - & $.59^{* *}$ & $.63^{* *}$ & -0.18 & $.66^{* *}$ & $.55^{* *}$ & 0.21 & $.47^{* *}$ & $.55^{* *}$ \\
\hline 6. GOALS T1 & - & - & - & - & - & $.61^{* *}$ & 0.09 & $.61^{* *}$ & $.41^{* *}$ & $.38^{*}$ & $.48^{*}$ & $.43^{*}$ \\
\hline 7. IMPUL T1 & - & - & - & - & - & - & 0.08 & $.70^{* *}$ & $.44^{* *}$ & 0.19 & $.35^{*}$ & $.51^{* *}$ \\
\hline 8. AWA T1 & - & - & - & - & - & - & - & -0.09 & $.11^{* *}$ & -0.09 & 0.06 & -0.08 \\
\hline 9. STRAT T1 & - & - & - & - & - & - & - & - & $.50^{* *}$ & 0.18 & $.46^{* *}$ & $.63^{* *}$ \\
\hline 10. CLA T1 & - & - & - & - & - & - & - & - & - & -0.16 & $.56^{* *}$ & $.44^{*}$ \\
\hline 11. HRV T2 & - & - & - & - & - & - & - & - & - & - & 0.08 & 0.03 \\
\hline 12. DEP T2 & - & - & - & - & - & - & - & - & - & - & - & $.51^{* *}$ \\
\hline 13. ANX T2 & - & - & - & - & - & - & - & - & - & - & - & - \\
\hline
\end{tabular}

${ }^{*} p<.05 ;{ }^{*} \mathrm{p} \leq .01 ; \mathrm{N} \mathrm{T1}=84 ; \mathrm{N}$ T2= 42; N T1xT2= 42. HRV= Heart rate variability; DEP= Depression; ANX= Anxiety; NONA= NONACCEPT; IMPUL= IMPULSE; AWA= AWARE; STRAT = STRATEGIES; CLA=CLARITY

\section{Moderation analyse}

Detailed results are reported in Table 3. A main effect of the total DERS score on depression was observed $(\beta=.28, t=2.37, p$ $\leq .05)$ controlling for depression at Time $1(\beta=.62, t=5.53, p \leq .01)$. The overall model explained $64 \%$ of depression variance $(\mathrm{F}(4,36)$ $=16.06, \mathrm{p} \leq .01$ ). No interaction effect totalDERSxHRV was found $(ß=.20, t=1.68, n s)$. The models including the subdimensions correlated with depression and anxiety at Time 2 (respectively $\mathrm{r}=$ $.75, \mathrm{p} \leq .01$ and $\mathrm{r}=.73, \mathrm{p} \leq .01$ ). HRV was not significantly associated with either the DERS scores or depression and anxiety scores at any time Table 2 .

Table 3: Moderation analyses

\begin{tabular}{|c|c|c|c|}
\hline \multicolumn{2}{|c|}{ Depression T2 } & \multicolumn{2}{|c|}{ Anxiety T2 } \\
\hline \multicolumn{2}{|c|}{ B (SE) } & \multicolumn{2}{|c|}{ B (SE) } \\
\hline Depression T1 & $.62(.11)^{* *}$ & Anxiety T1 & $.64(.15)^{* *}$ \\
\hline Total DERS T1 & $.28^{*}$ & Total DERS T1 & $.33(.13)^{*}$ \\
\hline HRV T1 & $-.11(.11)$ & HRV T1 & $-.17(.11)$ \\
\hline Total DERS T1 x HRV T1 & $.20(.12)$ & Total DERS T1 x HRV T1 & $.11(.13)$ \\
\hline $\mathrm{R}^{2}$ & $.64^{* *}$ & $\mathrm{R}^{2}$ & $.61^{* *}$ \\
\hline $\mathrm{F}$ & $16.06^{* *}$ & $\mathrm{~F}$ & $14.02^{* *}$ \\
\hline Depression $\mathrm{T} 1$ & $.67(.11)^{* *}$ & Anxiety $\mathrm{T} 1$ & $.71(.15)^{* *}$ \\
\hline NONACCEPT T1 & $.24(.12)^{*}$ & NONACCEPT T1 & $.25(.14)$ \\
\hline HRV T1 & $-.08(.11)$ & HRV T1 & $-.14(.11)$ \\
\hline NONACCEPT T1 x HRV T1 & $.13(.11)$ & NONACCEPT x HRV T1 & $.06(.11)$ \\
\hline $\mathrm{R}^{2}$ & $.63^{* *}$ & $\mathrm{R}^{2}$ & $.58^{* *}$ \\
\hline $\mathrm{F}$ & $15.57^{* *}$ & $\mathrm{~F}$ & $12.68^{* *}$ \\
\hline Depression T1 & $.64(.11)^{* *}$ & Anxiety T1 & $.78(.15)^{* *}$ \\
\hline GOALS T1 & $.24(.11)^{*}$ & GOALS T1 & $.12(.13)$ \\
\hline HRV T1 & $-.14(.12)$ & HRV T1 & $-.11(.13)$ \\
\hline GOALS T1 x HRV T1 & $.15(.12)$ & GOALS T1 x HRV T1 & $-.02(.13)$ \\
\hline $\mathrm{R}^{2}$ & $.63^{* *}$ & $\mathrm{R}^{2}$ & $.55^{* *}$ \\
\hline
\end{tabular}




\begin{tabular}{|c|c|c|c|}
\hline $\mathrm{F}$ & $15.07^{* *}$ & $\mathrm{~F}$ & $11.11^{* *}$ \\
\hline Depression $\mathrm{T} 1$ & $.70(.11)^{* *}$ & Anxiety T1 & $.73(.12)^{* *}$ \\
\hline IMPULSE T1 & $.21(.11)$ & IMPULSE T1 & $.33(.12)^{* *}$ \\
\hline HRV T1 & $-.02(.11)$ & HRV T1 & $-.09(.10)$ \\
\hline IMPULSE T1 x HRV T1 & $.05(.12)$ & IMPULSE T1 x HRV T1 & $.05(.11)$ \\
\hline $\mathrm{R}^{2}$ & $.61^{* *}$ & $\mathrm{R}^{2}$ & $.62^{* *}$ \\
\hline $\mathrm{F}$ & $13.94^{* *}$ & $\mathrm{~F}$ & $14.94^{* *}$ \\
\hline Depression T1 & $.74(.11)^{* *}$ & Anxiety $\mathrm{T} 1$ & $.88(.13)^{* *}$ \\
\hline AWARE T1 & $-.01(.11)$ & AWARE T1 & $.00(.11)$ \\
\hline HRV T1 & $-.02(.11)$ & HRV T1 & $-.12(.12)$ \\
\hline AWARE T1 x HRV T1 & $.01(.11)$ & AWARE T1 x HRV T1 & $.11(.11)$ \\
\hline $\mathrm{R}^{2}$ & $.57^{* *}$ & $\mathrm{R}^{2}$ & $.55^{* *}$ \\
\hline $\mathrm{F}$ & $11.98^{* *}$ & $\mathrm{~F}$ & $11.16^{* *}$ \\
\hline Depression T1 & $.68(.12)^{* *}$ & Anxiety T1 & $.62(.16)^{* *}$ \\
\hline STRATEGIES T1 & $.10(.12)$ & STRATEGIES T1 & $.31(.13) *$ \\
\hline HRV T1 & $-.05(.11)$ & HRV T1 & $-.14(.11)$ \\
\hline STRATEGIES T1 $\mathrm{x}$ HRV T1 & $.07(.09)$ & STRATEGIES T1 x HRV T1 & $-.04(.08)$ \\
\hline $\mathrm{R}^{2}$ & $.59^{* *}$ & $\mathrm{R}^{2}$ & $.60^{* *}$ \\
\hline $\mathrm{F}$ & $12.84^{* *}$ & $\mathrm{~F}$ & $13.59^{* *}$ \\
\hline Depression T1 & $.64(.13)^{* *}$ & Anxiety $\mathrm{T} 1$ & $.78(.15)^{* *}$ \\
\hline CLARITY T1 & $.21(.13)$ & CLARITY T1 & $.13(.13)$ \\
\hline HRV T1 & $.01(.11)$ & HRV T1 & $-.09(.11)$ \\
\hline CLARITY T1 x HRV T1 & $.12(.16)$ & CLARITY T1 x HRV T1 & $.02(.18)$ \\
\hline $\mathrm{R}^{2}$ & $.60^{* *}$ & $\mathrm{R}^{2}$ & $.55^{* *}$ \\
\hline $\mathrm{F}$ & $13.69^{* *}$ & $\mathrm{~F}$ & $11.13^{* *}$ \\
\hline
\end{tabular}

$\beta=$ Standardized beta coefficient $;$ SE $=$ Standard error

${ }^{*} p \leq .05,{ }^{* *} p \leq .01 \mathrm{HRV}=$ Heart rate variability; DEP= Depression; ANX= Anxiety; NONA= NONACCEPT; IMPUL= IMPULSE; AWA= AWARE; STRAT= STRATEGIES; CLA=CLARITY

\section{Mediation analyses}

A main effect of total DERS score on depression was found $(ß=$ $.24, \mathrm{t}=2.00, \mathrm{p} \leq .05)$ but no indirect effect of $\operatorname{HRV}(\beta=-.01,95 \% \mathrm{CI}=$ [-.10 to .02]), controlling for depression at Time $1(\beta=.62, t=5.37$, $\mathrm{p} \leq .01)$. The overall model explained $61 \%$ of depression variance ( $F$ $(3,37)=19.50, p \leq .01)$. No significant mediation effects using the subdimensions of the DERS were found (data not shown). A main effect of the total DERS score on anxiety was found $(B=.65, t=4.43$, $\mathrm{p}=<.05)$ but no indirect effect was found for HRV $(\beta=-.02,95 \%$ $\mathrm{CI}=[-.12$ to .01$])$ controlling for anxiety at Time $1(\beta=.65, \mathrm{t}=4.43$, $\mathrm{p} \leq .01)$. The overall model explained $60 \%$ of anxiety variance ( $\mathrm{F}$ $(3,37)=18.58, p \leq .01)$. No significant mediation effects using the subdimensions of the DERS were found (data not shown).

\section{Discussion}

The aim of this study was to examine the interaction between emotion regulation difficulties and HRV and their combined or individual effects on depression and anxiety symptoms persistence in CHD patients that have completed three month of cardiac rehabilitation. Two different models were tested in order to respect the mixed literature. The first one suggested that emotion regulation difficulties at Time 1 would predict persistence of depression and anxiety symptoms at Time 2 but that this relationship would be moderated by HRV's level at Time 1 . The second one hypothesized that emotion regulation difficulties at Time 1 would predict depression and anxiety persistence at Time 2 but that this relationship would be mediated by HRV's level at Time 1. Our results shown that emotion regulation difficulties predicted depression and anxiety persistence at 3-month followup even after controlling for depression or anxiety scores at baseline. These findings reinforce previous studies suggesting that emotion regulation difficulties are associated with depression and anxiety $[6,7,13,15]$ and furthermore, with the maintenance of these disorders $[10,9,14]$. The subdimensions NONACCEPT (nonacceptance of emotional responses) and GOALS (difficulties in engaging in goal-oriented behaviours when experience negative emotions) were also linked to the persistence of depression independently of depression at baseline. Non-acceptance of emotions has been already linked to depression [6] but to our knowledge, not with difficulties in maintaining goal-oriented behaviors. As some therapeutic interventions designed to reduce depressive symptoms imply to work on planned and organized goals it may be very difficult for the patients that exhibit a deficit in maintaining goal-oriented behaviors [42]. Previous researches 
have also shown an influence of emotion regulation strategies $[8,9]$ on depression as well as emotion clarity and awareness [9] but we did not replicate these results.

Regarding anxiety, the subdimensions IMPULSE (difficulties in controlling impulsive behaviours when experiencing negative emotions) and STRATEGIES (lack of strategies to regulate emotions) predicted the persistence of this trouble independently of the baseline level. This is consistent with [43] hypothesis that anxiety may be related to the inaccessibility of regulation strategies perceived as effective and the perceived inability to control behaviour in the face of distress. Furthermore, our results suggest that emotion regulation difficulties are linked to depression and anxiety but not in the same way as specific subdimensions of emotion regulation predicted the persistence of these disorders. Although we chose to rely on the theoretical approach suggesting that difficulties in emotion regulation impact depressive and anxious symptoms persistence $[13,14,15]$, it is of course more likely that this relationship is bidirectional. Indeed, it seems logical that depressed and anxious people have more difficulties to regulate their emotions as they are already in a distressed mood.

Finally, HRV did not predict either anxiety or depression persistence at 3-month follow-up. Many previous studies have reported associations between HRV and depression or anxiety $[44,35]$. However, some studies have also evidenced no relationship between depression and HRV $[45,46]$ or anxiety and HRV $[47,48]$. No associations either were found between HRV and emotion regulations difficulties at any time of our study contradictory to some previous research conducted on healthy individuals $[49,50]$. HRV is used as a peripheral measure of the prefrontal cortex's capacity to inhibit the amygdala [51] leading many scientists to consider HRV as a peripheral measure of emotion regulation [52]. A recent meta-analysis has confirmed this showing that HRV is linked to self-regulation top-down processes [26]. Furthermore, no moderation or mediation effect of HRV on the relationships between emotion regulation and mental health was observed. This is contradictory to previous studies $[53,54]$ showing a protective role of HRV on these relationships. However, all these studies do not rely on clinical populations suffering from cardiac diseases. To the extent of our knowledge, only one study focused on HRV and emotional processes in CHD patients and no relationship between HRV and emotional sensitivity appeared although each of these variables was predictive of cardiac death at 8-years follow-up [55].

Our study has several limitations worth noting. First, a dropout of $50 \%$ was observed from Time 1 to Time 2 leading to a reduced sample. We do not have informations on the reasons leading these patients to stop their cardiac rehabilitation as the analyses were not significant. Thus, we cannot exclude a bias in the resulting sample (i.e. patients more motivated, less depressed or anxious, more concerned about their health, in better physical condition/ health,...). Second, our sample was composed of both males and few females. Although we did not observe significant differences on dependent variables between genders, it could impact the generalizability of our results. Indeed, gender differences in emotion regulation strategies have been previously reported in the literature [56].

\section{Conclusion}

Our study is the first that highlights the predictive value of emotion regulation difficulties on the persistence of depression and anxiety symptoms at the end of cardiac rehabilitation of CHD patients. Because depression and anxiety negatively impacts the prognostic of these patients [57], it seems crucial to understand the factors that account for the maintenance of these troubles to be able to modify them. In this idea, it would be interesting to evaluate if emotions-management training could help CHD patients by reducing depression and anxiety symptoms better than existing psychological interventions such a stress-management training [56].

\section{Acknowledgement}

None.

\section{Conflict of Interest}

No conflict of interest.

\section{References}

1. Gross JJ (1998) The emerging field of emotion regulation: an integrative review. Review of General Psychology 2(3): 271-299.

2. Gratz KL, Roemer E (2004) Multidimensional Assessment of Emotion Regulation and Dysregulation: Development, Factor Structure, and Initial Validation of the Difficulties in Emotion Regulation Scale. Journal of Psychopathology and Behavioral Assessment 26(1): 41-54.

3. Gross JJ, and Jazaieri H (2014) Emotion, emotion regulation, and psychopathology: an affective science perspective. Clinical Psychological Science 2: 387-401.

4. Aldao A, Nolen-Hoeksema S, Schweizer S (2010) Emotion-regulation strategies across psychopathology: A meta-analytic review. Clinical psychology review 30(2): 217-237.

5. Berking M, Wupperman P (2012) Emotion regulation and mental health: recent findings, current challenges, and future directions. Current Opinion in Psychiatry 25: 128-134.

6. Campbell-Sills L, Barlo DH, Brown TA, Hofmann SG (2006) Acceptability and suppression of negative emotion in anxiety and mood disorders. Emotion 6: 587-595.

7. Garnefski N, Kraaij V (2006) Relationships between cognitive emotion regulation strategies and depressive symptoms: A comparative study of five specific samples. Personality and Individual Differences 40: 16591669.

8. Gross JJ, John OP (2003) Individual differences in two emotion regulation processes: Implications for affect, relationships, and well-being. Journal of Personality and Social Psychology 85: 348-362.

9. Rude SS, McCarthy CT (2003) Emotional functioning in depressed and depression- vulnerable college students. Cognition and Emotion 17: 799-806.

10. Ehring T, Fischer S, Schnülle J, Bösterling A, Tuschen-Caffier B, et al. (2008) Characteristics of emotion regulation in recovered depressed versus never depressed individuals. Personality and Individual Differences 44(7): 1574-1584.

11. Kraaij V, Pruymboom E, Garnefski N (2002) Cognitive coping and depressive symptoms in the elderly: a longitudinal study. Aging \& Mental Health 6: 275-281. 
12. Kring AM, Werner KH (2004) Emotion regulation and psychopathology. In P Philippot, RS Feldman (Eds.), The regulation of emotion pp. 359385.

13. Mennin DS, Heimberg RG, Turk CL, Fresco DM (2005) Preliminary evidence for an emotion regulation deficit model of generalized anxiety disorder. Behaviour Research and Therapy 43: 1281-1310.

14. Salters-Pedneault K, Roemer L, Tull MT, Rucker L, Mennin DS, et al. (2006) Evidence of broad deficits in emotion regulation associated with chronic worry and generalized anxiety disorder. Cognitive Therapy and Research 30(4): 469-480.

15. Tull MT, Stipelman BA, Salters-Pedneault K, Gratz KL (2009) An examination of recent nonclinical panic attacks, panic disorder, anxiety sensitivity, and emotion regulation difficulties in the prediction of generalized anxiety disorder in an analogue sample. Journal of Anxiety Disorders 23: 275-282.

16. Eng HS, Yean LC, Das S, Letchmi S, Yee KS (2011) Anxiety and depression in patients with coronary heart disease: a study in a tertiary hospital. Iranian journal of medical sciences 36(3): 201-206.

17. Roest AM, Martens EJ, Denollet J, De Jonge P (2010) Prognostic association of anxiety post myocardial infarction with mortality and new cardiac events: a meta-analysis. Psychosomatic medicine 72(6): 563-569.

18. van Melle JP, De Jonge P, Spijkerman TA, Tijssen JG, Ormel J, et al. (2004) Prognostic association of depression following myocardial infarction with mortality and cardiovascular events: a meta-analysis. Psychosomatic Medicine 66(6): 814-822.

19. Appelhans BM, Luecken, LJ (2006) Heart rate variability as an index of regulated emotional responding. Review of general psychology 10(3): 229-240

20. Beauchaine TP, Thayer JF (2015) Heart rate variability as a transdiagnostic biomarker of psychopathology. International Journal of Psychophysiology 98(2 Pt2): 338-350.

21. Thayer JF, Lane RD (2000) A model of neurovisceral integration in emotion regulation and dysregulation. Journal of Affective Disorders 61: $201-216$

22. Buccelletti E, Gilardi E, Scaini E, Galiuto L, Persiani R, et al. (2009) Heart rate variability and myocardial infarction : systemac literature review and metanalysis. European Review for Medical and Pharmacological Sciences 13: 299-307.

23. Cameron O (1996) Depression increases post-MI mortality: How? Psychosomatic Medicine 58: 111-112.

24. Thayer JF, Lane RD (2007) The role of vagal function in the risk for cardiovascular disease and mortality. Biological Psychiatry 74: 224-242.

25. Porges SW (2001) The polyvagal theory: Phylogenetic substrates of a social nervous system. International Journal of Psychophysiology 42: 123-146

26. Holzman JB, Bridgett DJ (2017) Heart rate variability indices as biomarkers of top-down self-regulatory mechanisms: a meta-analytic review. Neuroscience \& Biobehavioral Reviews 74(Pt A): 233-255.

27. Monteiro S, Monteiro B, Candida M, Adler N, Campos C, et al. (2016) Association between depression severity and executive functioning in late-life depression: a systematic review. Medical Express 3(6).

28. De Raedt R, Koster EH, Joormann J (2010) Attentional control in depression: A translational affective neuroscience approach. Cognitive, Affective, \& Behavioral Neuroscience 10(1): 1-7.

29. Ajilchi B, Nejati V (2017) Executive functions in students with depression, anxiety, and stress symptoms. Basic and clinical neuroscience 8(3): 223232.

30. Carnevali L, Thayer JF, Brosschot JF, Ottaviani C (2018) Heart rate variability mediates the link between rumination and depressive symptoms: A longitudinal study. International Journal of Psychophysiology 131: 131-138.

31. Gillie BL, Vasey MW, Thayer JF (2014) Heart rate variability predicts control over memory retrieval. Psychological Science 25(2): 458-465.
32. Batselé E, Stefaniak N, \& Fantini-Hauwel C (2019) Resting heart rate variability moderates the relationship between trait emotional competencies and depression. Personality and Individual Differences 138: 69-74.

33. Ode S, Hilmert CJ, Zielke DJ, Robinson MD (2010) Neuroticism's importance in understanding the daily life correlates of heart rate variability. Emotion 10(4): 536-543.

34. Conraads VM, Deaton C, Piotrowicz E, Santaularia N, Tierney S, et al. (2012) Adherence of heart failure patients to exercise: barriers and possible solutions. European journal of heart failure 14(5): 451-458

35. Kemp AH, Quintana DS, Gray MA, Felmingham KL, Brown K, et al. (2010) Impact of Depression and Antidepressant Treatment on Heart Rate Variability: A Review and Meta-Analysis. Biological Psychiatry 67: 10671074.

36. Zigmond AS, Snaith RP (1983) The hospital anxiety and depression scale. Acta Psychiatrica Scandinavica 67: 361-370

37. Task Force of The European Society of Cardiology and The North American Society of Pacing and Electrophysiology (1996) Heart rate variability: Standards of measurement, physiological interpretation, and clinical use. European Heart Journal 17: 354-381.

38. Tarvainen MP, Niskanen JP, Lipponen JA, Ranta-Aho PO, Karjalainen PA (2014) Kubios HRV - heart rate variability analysis software. Comput Methods Programs Biomed 113(1): 210-220.

39. Hill LK, Siebenbrock A (2009) Are all measures created equal? Heart rate variability and respiration. Biomedical Sciences Instrumentation 45: 7176.

40. Ellis RJ, Sollers Iii JJ, Edelstein EA, Thayer JF (2008) Data transforms for spectral analyses of heart rate variability. Biomedical Sciences Instrumentation 44: 392-397.

41. IBM Corp Released (2017) IBM SPSS Statistics for Windows, Version 25.0. Armonk, NY: IBM Corp.

42. Lejuez CW, Hopko DR, Hopko SD (2001) A brief behavioral activation treatment for depression: Treatment manual. Behavior Modification 25(2): 255-286

43. Mennin DS, Heimberg RG, Turk CL, Fresco DM (2002) Applying an emotion regulation framework to integrative approaches to generalized anxiety disorder. Clinical Psychology: Science \& Practice 9: 85-90.

44. Brunoni AR, Kemp AH, Dantas EM, Goulart AC, Nunes MA (2013) Heart rate variability is a trait marker of major depressive disorder: evidence from the sertraline vs. electric current therapy to treat depression clinical study. International Journal of Neuropsychopharmacology 16(9): 1937-1949.

45. Agelink MW, Majewski T, Wurthmann C, Postert T, Linka T, et al. (2001) Autonomic neurocardiac function in patients with major depression and effects of antidepressive treatment with nefazodone. Journal of Affective Disorders 62(3): 187-198.

46. Moser M, Lehofer M, Hoehn-Saric R, McLeod DR, Hildebrandt G, et al. (1998) Increased heart rate in depressed subjects in spite of unchanged autonomic balance. Journal of affective disorders 48(2-3): 115-124.

47. Einvik G, Hrubos-Strom H, Randby A, Nordhus IH, Somers VK, et al. (2011) Major depressive disorder, anxiety disorders, and cardiac biomark- ers in subjects at high risk of obstructive sleep apnea. Psychosomatic Medicine 73(5): 378-84.

48. Hammel JC, Smitherman TA, McGlynn FD, Mulfinger AM, Lazarte AA, et al. (2011) Vagal influence during worry and cognitive challenge. Anxiety, Stress, \& Coping 24(2): 121-136.

49. Visted E, Sørensen L, Osnes B, Svendsen JL, Binder PE, et al. (2017) The association between self-reported difficulties in emotion regulation and heart rate variability: the salient role of not accepting negative emotions. Frontiers in Psychology 8: 328.

50. Williams DP, Cash C, Rankin C, Bernardi A, Koenig J, et al. (2015) Resting heart rate variability predicts self-reported difficulties in emotion regulation: a focus on different facets of emotion regulation. Frontiers in Psychology 6: 261. 
51. Thayer JF, Ahs F, Fredrikson M, Sollers JJ, Wager TD, et al. (2012) A metaanalysis of heart rate variability and neuroimaging studies: implications for heart rate variability as a marker of stress and health. Neuroscience \& Biobehavioral Reviews 36(2): 747-756.

52. Johnstone T, Walter H (2014) The neural basis of emotion dysregulation, in Handbook of Emotion Regulation, 2nd Edn, ed. JJ Gross (New York, NY: The Guilford Press): 58-75.

53. Fantini-Hauwel C, Gois C, Stefaniak N, Batselé E (2019) Emotion regulation difficulties are not always associated with negatives outcomes: the buffer effect of cardiac vagal control. Article submitted in British Journal of Health Psychology.

54. Carpeggiani C, Emdin M, Bonaguidi F, Landi P, Michelassi C, et al. (2005) Personality traits and heart rate variability predict long-term cardiac mortality after myocardial infarction. European Heart Journal 26(16): 1612-1617

55. Nolen-Hoeksema S, Aldao A (2011) Gender and age differences in emotion regulation strategies and their relationship to depressive symptoms. Personality and Individual Differences 51(6): 704-708.

56. Whalley B, Rees K, Davies P, Bennett P, Ebrahim S, et al. (2011) Psychological interventions for coronary heart disease. Cochrane Database of Systematic Reviews (8).

57. Gross JJ (Ed.). (2013) Handbook of emotion regulation. Guilford publications. 